\title{
Recent Developments in Fluctuation X-ray Scattering at X-FELs
}

\author{
K. Pande ${ }^{1,2}$, J. J. Donatelli ${ }^{2,3}$, C.Gati ${ }^{4}$, M. Hunter ${ }^{4}$, R. A. Kirian ${ }^{5}$, P. H. Zwart ${ }^{1,2}$
}

${ }^{1}$ Molecular Biophysics and Integrated Bio-Imaging Division, LBNL, Berkeley, CA, USA (kpande@lbl.gov)

${ }^{2}$ Center for Advanced Mathematics for Energy Research Applications, LBNL, Berkeley, CA, USA

${ }^{3}$ Department of Mathematics, Computational Research Division, LBNL, Berkeley, CA, USA

${ }^{4}$ SLAC National Accelerator Laboratory, Menlo Park, CA, USA

${ }^{5}$ Arizona State University, Tempe, AZ, USA

Fluctuation X-ray scattering (FXS) is a biophysical technique that overcomes the low datato-parameter ratios encountered in traditional X-ray scattering methods used for studying noncrystalline samples [1]. In an FXS experiment solution scattering data are collected from particles in solution using ultrashort X-rays of pulse lengths shorter than the rotational diffusion time of the particles. The resulting data contains angularly varying information that yields structures with a greater level of detail than those obtained using tradition SAXS [2].

After successful application of FXS to single-particle data [3], we have recently demonstrated the experimental feasibility of this technique on data from an ensemble of PBCV-1 virus particles in solution $[4,5]$. We found that by using advanced noise-filtering methods the required number of images to obtain decent correlation data is far lower than originally expected, thereby reducing the required data collection time to less than a few minutes. In this talk I will outline the data processing techniques for analysis of FXS data, and present an assessment of the effect of concentration and various sources of noise on experimental data.

This research was supported by the Advanced Scientific Computing Research and the Basic Energy Sciences programs of the Office of Science of the US Department of Energy (DOE), and the National Institute of General Medical Sciences of the National Institutes of Health (NIH).

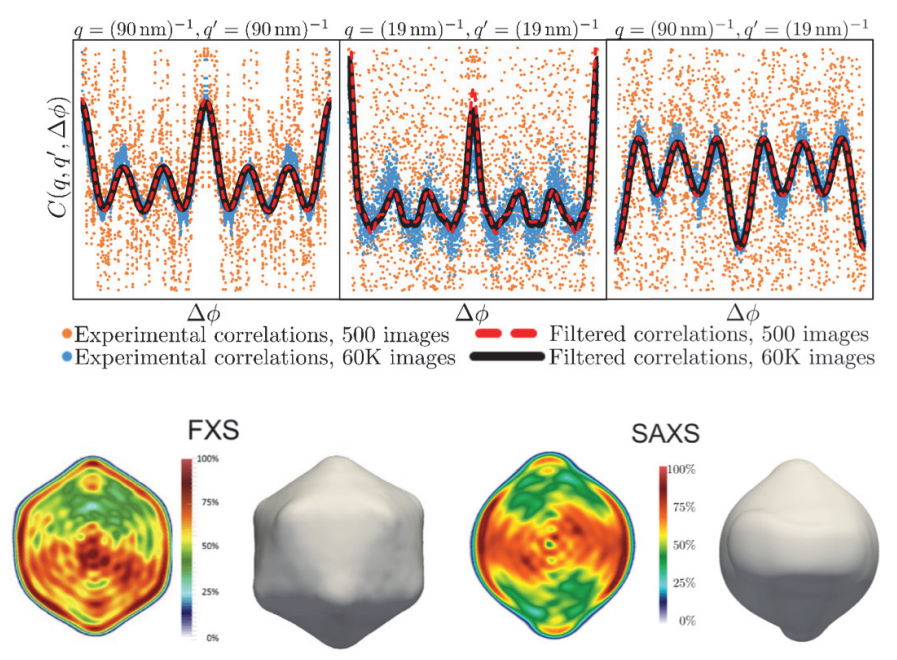

\section{References}

[1] Kam Z, Koch MH, Bordas J. (1981). PNAS 78 (6), 3559-62.

[2] Donatelli JJ, Zwart PH, Sethian JA. (2015). PNAS 112 (33), 10286-10291.

[3] Kurta RP, et al. (2017). Phys Rev Lett., 119 (15), 158102.

[4] Pande K, Donatelli JJ, Malmerberg E, Foucar L, Bostedt C, Schlichting I, Zwart PH. (2018). PNAS, 115 (46), 11772-11777.

[5] Pande K, et al. (2018). Scientific Data, 5, 180201. 